\title{
Evolución de las licencias por enfermedad grave del niño(a) menor de un año 2004-2008: certezas e interrogantes
}

\author{
IRIS DELGADO B. ${ }^{1, a}$, MACARENA HIRMAS A. ${ }^{1, b}$, FLORENCIA PRIETO U. ${ }^{2, c}$
}

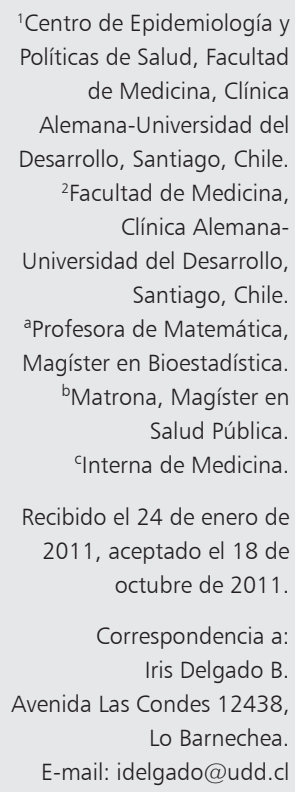

\section{Sick leaves motivated by severe diseases of children of less than one year of age}

Background: The increase in expenses of the health care system caused by sick leaves, especially those granted to mothers with children of less than one year of age with severe diseases, is a topic of concern. Aim: To describe the rates of sick leaves granted to mothers with children of less than one year of age with severe diseases from 2004 to 2008. Material and Methods: Analysis of databases containing information about sick leaves coming from Chilean public and private health care subsystems. Leaves granted to mothers with sick children of less than one year were specifically analyzed in terms of days off work and the amount of monetary benefits. Results: A sustained increase, ranging from 20 to $120 \%$ in the number of sick leaves motivated by diseases of children of less than one year, was observed. Thirty four percent of maternity leaves concentrate immediately after finishing the legal period (24 weeks after birth) and almost 60\% of them occured within the first 6 months after birth. The most frequent diagnoses that motivated the higher number of leaves were gastroesophageal reflux and bronchitis. Conclusions: There is probably a bad use of the benefit in a percentage of leaves. The benefit is also regressive since it favors mostly mothers of a better socioeconomic condition. The law that will extend the postnatal maternity leave will solve in part these problems.

(Rev Med Chile 2012; 140: 30-38).

Key words: Maternal-child nursing; Maternity leave; Sick leave.
E n Chile, la tendencia al aumento en el uso de licencias médicas es evidente en los últimos años. Este aumento ha estado sometido a una amplia discusión principalmente a causa de los costos que ello significa, ya que implica una carga creciente para los sistemas de salud pública y privados, que se traduce en una reducción de los recursos disponibles para la cobertura del resto de la atención de salud ${ }^{1}$.

La licencia por enfermedad grave de niño menor de un año (EGNMA) es un subsidio para la mujer que trabaja recibiendo el $100 \%$ de la remuneración o renta imponible, con un tope de 60 UF mensuales por el tiempo en que se vea obligada a estar ausente del trabajo por alguna enfermedad grave del niño menor a un año. Las licencias pre- post natal y por EGNMA representan casi el 90\% del total de licencias médicas No Curativas².

El financiamiento de este subsidio es independiente de la afiliación previsional y condición socioeconómica de la trabajadora y se realiza únicamente con aportes fiscales mediante el Fondo Único de Prestaciones Familiares y Subsidios de Cesantía, cuya administración financiera, control y tuición están a cargo de la Superintendencia de Seguridad Social ${ }^{3,4}$.

Las licencias por EGNMA han aumentado significativamente entre los años 2004 y 2008, tanto para las cotizantes del seguro público, Fondo Nacional de Salud (FONASA) como para las cotizantes de Instituciones de Salud Previsional (ISAPRE). En 2004 se emitieron por esta causa 18 
licencias por cada 100 cotizantes mujeres ( 226.255 licencias). En el 2008 esta tasa alcanzó a 32 por 100 cotizantes (476.321 licencias). Es decir, la tasa de uso de licencias aumentó en $80 \%$ en el período 2 .

En relación al gasto, según datos de Superintendencia de Salud, el año 2009 el Estado destinó $\$ 82.155$ millones al pago de subsidios por EGNMA. Al compararlo con el año 2001 se muestra un aumento del 109\%, (\$37.132 millones), ambos en pesos de diciembre $2009^{1}$.

Dado los indicadores de morbimortalidad infantil que posicionan a Chile en un lugar avanzado en materia sanitaria, surge la interrogante ¿Por qué hay un aumento en el número y tasa de licencias por EGNMA, sin haber un incremento significativo en el número de nacidos vivos, ni en la morbilidad y mortalidad infantil?, algunas de las posibles respuestas son: a) Hay un aumento de enfermedades específicas que afectan principalmente a los menores de un año; b) El subsidio se está usando para extender la lactancia materna o el reposo maternal; c) El diseño actual del subsidio permite que existan incentivos para un sobreuso ${ }^{3,5-9}$.

Los objetivos de este estudio son: describir las características asociadas al aumento en el uso y al gasto de las licencias por EGNMA; y comparar el uso y el gasto entre el sistema público y el privado en el período 2004 al 2008.

Este estudio aporta evidencia que contribuirá a la definición de políticas públicas que permitirán controlar, en caso de existir, el uso inadecuado de este subsidio y por otro lado, aportar con información para el diseño de estrategias destinadas a limitar el aumento del número de licencias y del gasto, sin afectar el buen uso de este derecho de los trabajadores y protección de la maternidad.

\section{Material y Método}

Estudio observacional basado en el análisis de datos secundarios, correspondientes al universo de licencias por EGNMA emitidas a las madres o padres entre los años 2004 y 2008 para la población perteneciente al FONASA e ISAPRE.

Las bases de datos fueron proporcionados por FONASA para la población perteneciente al sistema público, y por Superintendencia de Salud para la población de cotizante del sistema privado.

\section{Universo y unidad de análisis de la información}

Para FONASA e ISAPRE se analizó el universo de licencias médicas.

La información contenida en estas bases de datos corresponde a las variables del formulario de licencias médicas, que incluye: variable diagnóstico por el cual se emite la licencia codificada de acuerdo a la Clasificación Internacional de Enfermedades versión 10 (CIE-10); los días de licencia aprobados y pagados al cotizante y montos a cancelar para cada licencia. Se trabajó de manera anonimizada, asignando un número correlativo para cada individuo, repitiendo el número para las licencias correspondientes a una misma persona en cada año.

Se trabajó con dos unidades de análisis, la primera el "evento licencia" y la segunda "persona que solicita una o más licencias" durante el año.

\section{Análisis estadístico de la información}

a) Utilización de licencias médicas, para ambos universos (FONASA e ISAPRE) se calcularon:

1. Tasa de intensidad: representa el número de licencias emitidas por cada 100 cotizantes en edad fértil.

2. Duración promedio: representa el número promedio de días otorgados por cada licencia EGNMA.

3. Tasa de incapacidad laboral (TIL): corresponde al cociente entre días pagados por trabajadora debido a Licencias Médicas por EGNMA y el total de mujeres cotizantes en edad fértil.

4. Distribución por grupos de diagnóstico y causas específicas total y por edad del niño.

\section{b) Evolución del gasto}

En el caso de FONASA, se generó la variable "Total Monto Subsidio Incapacidad Laboral (SIL)" que incluye: Monto SIL; aporte previsional; aporte salud; aporte desahucio y subsidio de cesantía. Para las licencias de ISAPRE la base de datos incluye la variable Gasto Total SIL. Los montos fueron actualizados al valor peso diciembre año 2009.

\section{Resultados}

El universo de mujeres cotizantes en edad fértil (15 a 49 años) de FONASA el año 2008 era 1.127.244, que representa el 76\% del total, con un aumento del $18 \%$ en el período observado. Para 
ISAPRE este universo era de 349.557 cotizantes con un incremento del $11 \%$ entre los años 2004 y 2008.

El total de licencias médicas por EGNMA solicitadas en el período, para FONASA fue 1.127.145 requeridas por 240.231 mujeres. Para ISAPRE fue 523.314 solicitadas por 88.189 mujeres.

La edad promedio de las mujeres de FONASA que hicieron uso de licencias por EGNMA fue 29 años (DE $\pm 5,7)$, mientras que en ISAPRE fue 32 años $(\mathrm{DE} \pm 4,5)$.

El número de licencias por EGNMA solicitadas por hombres, fue pequeño, destacándose que para FONASA el aumento en el período fue de 158 a 1.114 licencias. En ISAPRE el aumento fue menor, de 49 a 89 licencias.

\section{Tasa de intensidad de uso}

La tasa de intensidad de uso de licencias por EGNMA aumentó en ambos sistemas. Para FONASA el aumento en términos porcentuales fue de $120 \%$, para ISAPRE fue $22 \%$. A pesar del importante aumento de FONASA en todo el período, la tasa de intensidad de uso fue mayor para las mujeres de ISAPRE (Figura 1), en otras palabras fueron las mujeres del sistema privado las que hicieron más uso de las licencias por EGNMA.

\section{Duración promedio de licencias}

La duración promedio del número de días aumentó en ambos sistemas. Al igual que en la tasa de intensidad de uso, la extensión de las licencias por EGNMA fue mayor para las mujeres de ISAPRE en todo el período. El incremento para ISAPRE fue de $10,6 \%$, mientras que para FONASA fue de $17,1 \%$ (Figura 2).

\section{Tasa de incapacidad laboral (TIL)}

La TIL aumentó en ambos subsistemas, siendo mayor para ISAPRE que FONASA en todo el período, no obstante que el porcentaje de aumento fue mayor para la población afiliada al sistema público (122\%), que para ISAPRE dicho incremento fue de $43 \%$ (Figura 3 ).

\section{Tipos de diagnósticos}

Los dos principales grupos de diagnósticos por los cuales se solicitó licencia EGNMA para FONASA e ISAPRE, correspondía a enfermedades del aparato digestivo (K00-K99) y enfermedades del aparato respiratorio (J00-J99).

El análisis por causas específicas, mostró que las principales patologías que originaban las licencias correspondía a reflujo (k21); bronquitis (J20); bajo peso al nacer (P07) y diarrea (A09), las dos primeras representaron más de $75 \%$ tanto para FONASA e ISAPRE (Tabla 1).

En ambos subsistemas la categoría "Otros" estuvo representada principalmente por neumonías (J18); otras enfermedades obstructivas crónicas (J44); rinofaringitis y faringitis agudas ( J00 y J02).

La tendencia observada en el período mostró que el diagnóstico de reflujo aumentó de $34 \%$ a $51 \%$ en FONASA, mientras que la bronquitis disminuyó desde 35\% a 22\%(Figura 4).

Para los niños(as) de madres pertenecientes a ISAPRE se observa la misma situación descrita para FONASA. Es decir, aumentó el reflujo y disminuye la bronquitis (Figura 4).

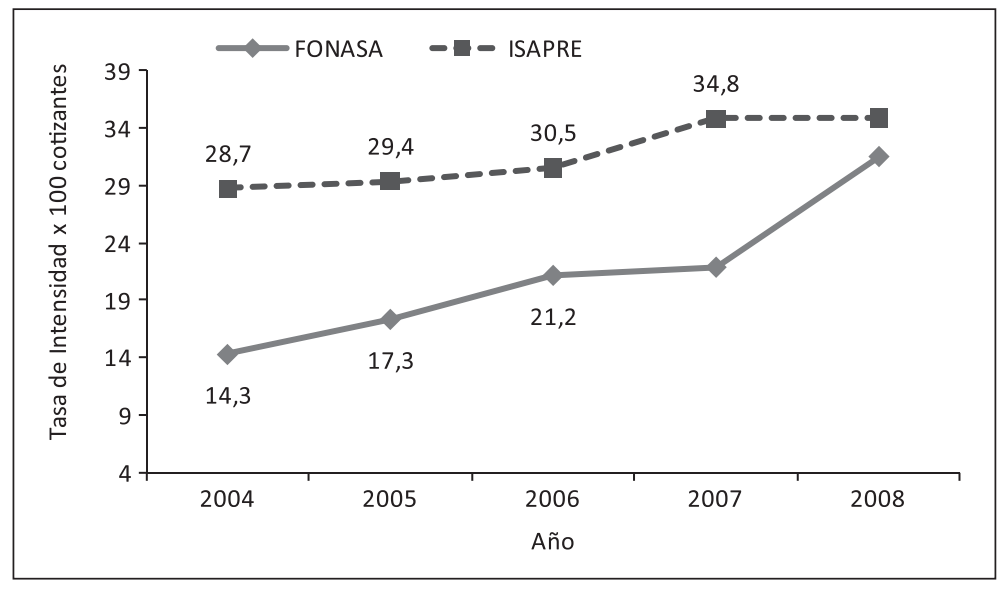

Figura 1. Tasa de Intensidad de uso por licencias EGNMA, según FONASA e ISAPRE, años 2004-2008. Fuente: Elaboración propia en base a datos de FONASA y Superintendencia de Salud. * Denominador: Mujeres cotizantes en edad fértil (15 a 49 años). 


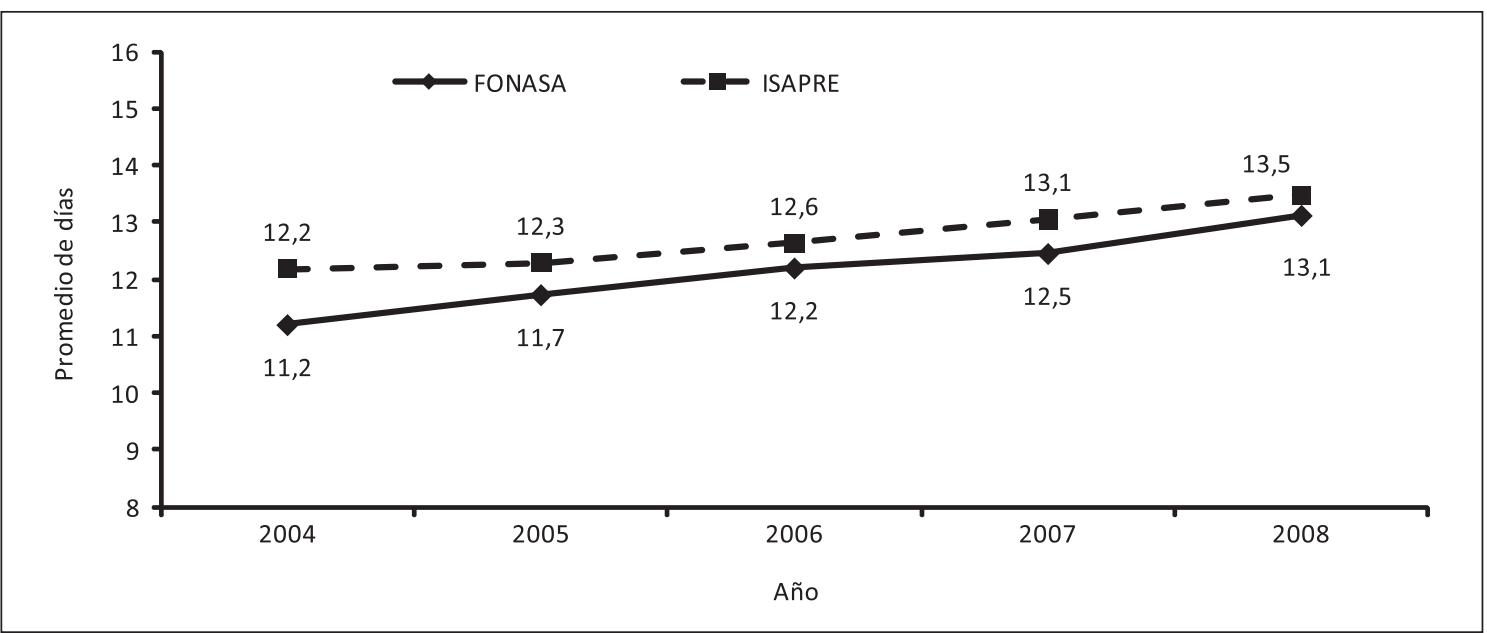

Figura 2. Duración promedio del número días otorgado de licencias por EGNMA, según FONASA e ISAPRE, años 2004-2008. Fuente: Elaboración propia en base a datos de FONASA y Superintendencia de Salud.

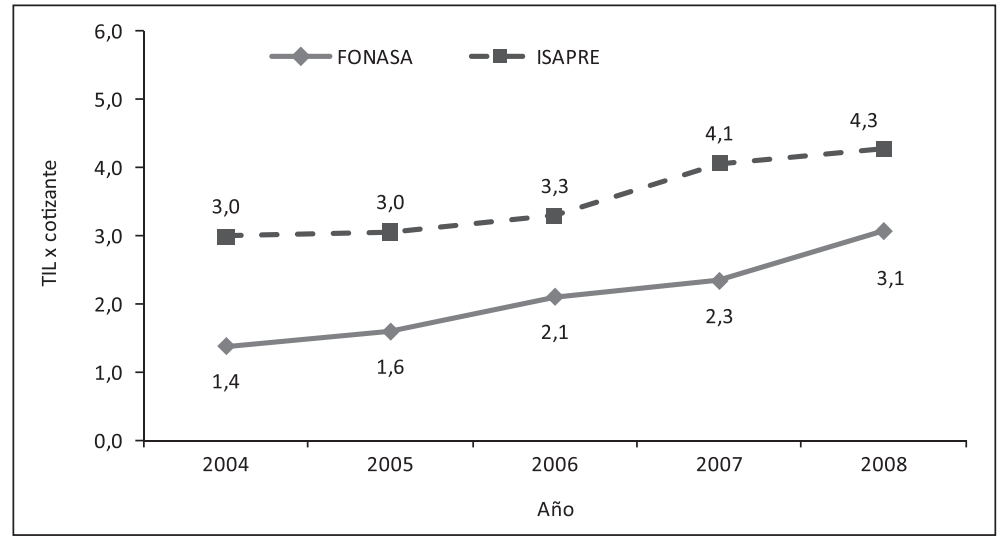

Figura 3. Tasa de Incapacidad Laboral por licencias EGNMA, según FONASA e ISAPRE, años 2004-2008. Fuente: Elaboración propia en base a datos de FONASA y Superintendencia de Salud. *Denominador: Mujeres cotizantes en edad fértil (15 a 49 años).

Tabla 1. Distribución porcentual de las licencias por EGNMA según causas específicas, para FONASA e ISAPRE, años 2004-2008

\begin{tabular}{|lrrrrrrrrrr|}
\hline Causa específica & \multicolumn{9}{c}{ FONASA } & \multicolumn{4}{c}{ ISAPRE } \\
& $\mathbf{2 0 0 4}$ & $\mathbf{2 0 0 5}$ & $\mathbf{2 0 0 6}$ & $\mathbf{2 0 0 7}$ & $\mathbf{2 0 0 8}$ & $\mathbf{2 0 0 4}$ & $\mathbf{2 0 0 5}$ & $\mathbf{2 0 0 6}$ & $\mathbf{2 0 0 7}$ & $\mathbf{2 0 0 8}$ \\
\hline Reflujo & 33,5 & 35,3 & 43,5 & 48,8 & 50,3 & 45,1 & 48,7 & 51,6 & 54,5 & 58,0 \\
Bronquitis & 35,3 & 29,6 & 26,5 & 25,5 & 21,6 & 18,6 & 17,8 & 17,0 & 15,9 & 14,3 \\
\hline Bajo peso al nacer & 1,5 & 1,2 & 1,2 & 1,0 & 1,0 & 0,7 & 1,0 & 1,1 & 1,1 & 1,2 \\
\hline Diarrea & 1,6 & 1,6 & 2,0 & 1,6 & 1,6 & 0,8 & 1,1 & 1,1 & 0,9 & 0,8 \\
\hline Otros & 28,2 & 32,3 & 26,8 & 23,0 & 25,4 & 34,8 & 31,4 & 29,1 & 27,5 & 25,6 \\
\hline Total & 100 & 100 & 100 & 100 & 100 & 100 & 100 & 100 & 100 & 100 \\
\hline
\end{tabular}

Fuente: Elaboración propia en base a datos de FONASA y Superintendencia de Salud. 


\section{Licencia EGNMA y edad del niño(a)}

$\mathrm{Al}$ analizar la edad del niño(a) en la cual la madre pide licencia por EGNMA se aprecia que una de cada tres licencias $(34,2 \%)$ era solicitada inmediatamente después de terminado el período de post-natal y más de la mitad (57,9\%) durante los 3 meses siguientes al término de la licencia postnatal. Se dispuso de esta información sólo para FONASA (Figura 5).

\section{Causa específica de las licencias y edad del niño(a)}

Inmediatamente terminado el posnatal, aproximadamente 5 de cada 10 licencias eran por reflujo. Entre los 4 y 9 meses de edad del niño, el reflujo seguía siendo la causa principal. En edades cercanas al año, la bronquitis adquirió relevancia como diagnóstico para las licencias por EGNMA (Figura 6).

\section{Evolución del gasto por cotizante}

El análisis de las cifras del gasto comparando entre seguro público y privado muestra importantes hallazgos. El promedio del gasto por cada mujer cotizante de ISAPRE en el año 2004 fue de $\$ 57.690$, mientras que para las mujeres de FONASA este monto alcanzó sólo a \$ 14.628 promedio, reflejando una brecha de 3,9 veces a favor de las cotizantes del seguro privado. En otras palabras, esto significa que $76 \%$ de la mujeres cotizantes que pertenecían al sistema público, se llevaron sólo $47,8 \%$ del monto total, mientras que en $23,9 \%$ de las mujeres cotizantes de ISAPRE se gastó 52\% del gasto total del subsidio de licencias medicas por EGNMA entre los años 2004 al 2008.

El aumento del gasto fue bastante homogéneo para ambos subsistemas, $51 \%$ para FONASA y $50 \%$ para ISAPRES, en otras palabras la brecha de desigualdad se ha mantenido inalterable (Figura 7$)$.

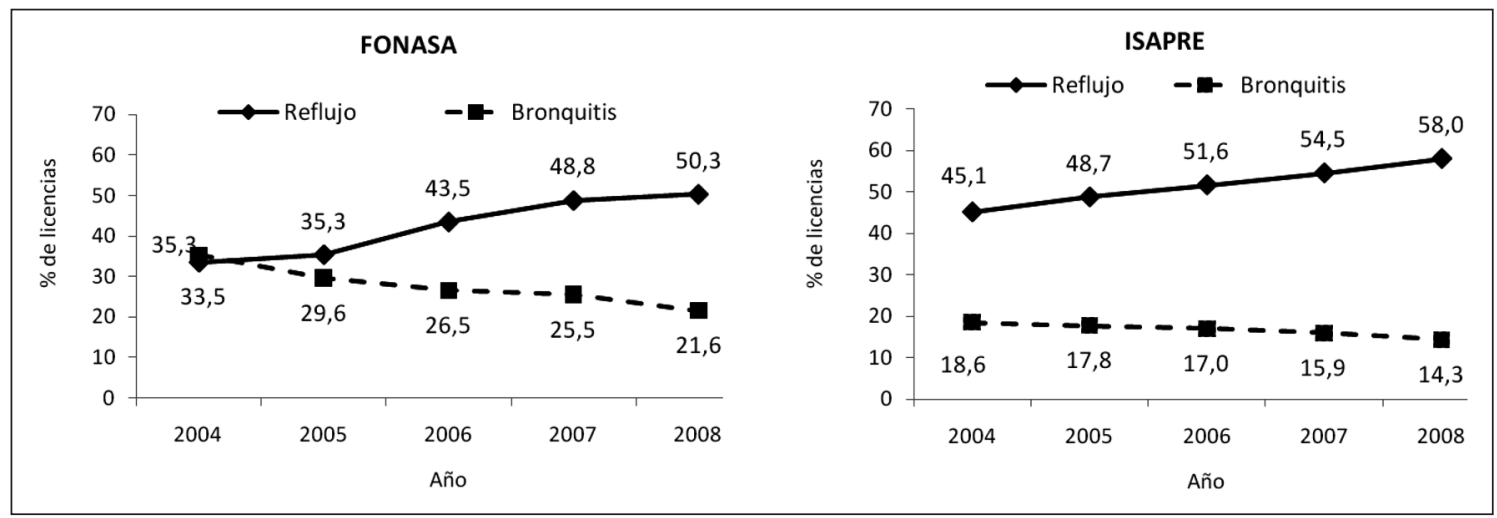

Figura 4. Evolución del reflujo y la bronquitis como causas de las licencias por EGNMA según FONASA e ISAPRE, años 2004 2008. Fuente: Elaboración propia en base a datos de FONASA y Superintendencia de Salud.

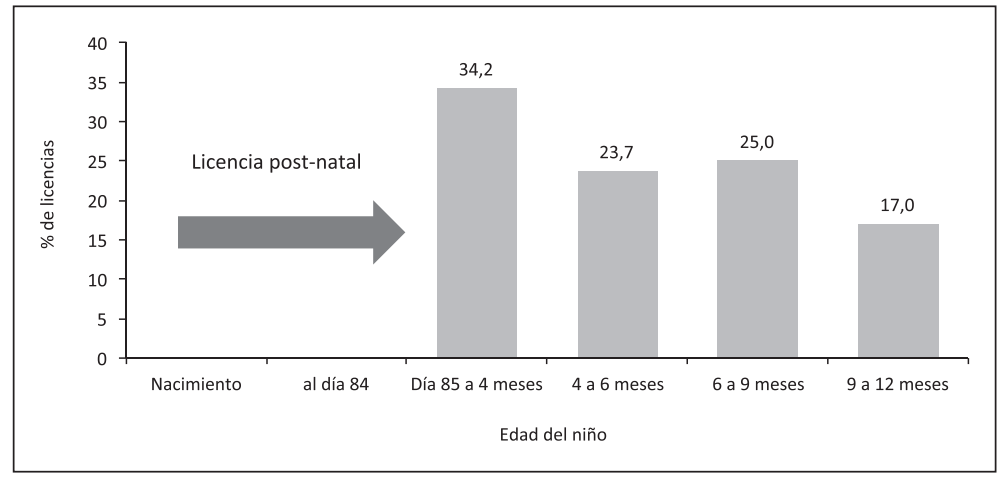

Figura 5. Porcentaje de licencias según edad del niño(a) en que se solicita la licencia por EGNMA, para FONASA, años 2004-2008. Fuente: Elaboración propia en base a datos de FONASA. 

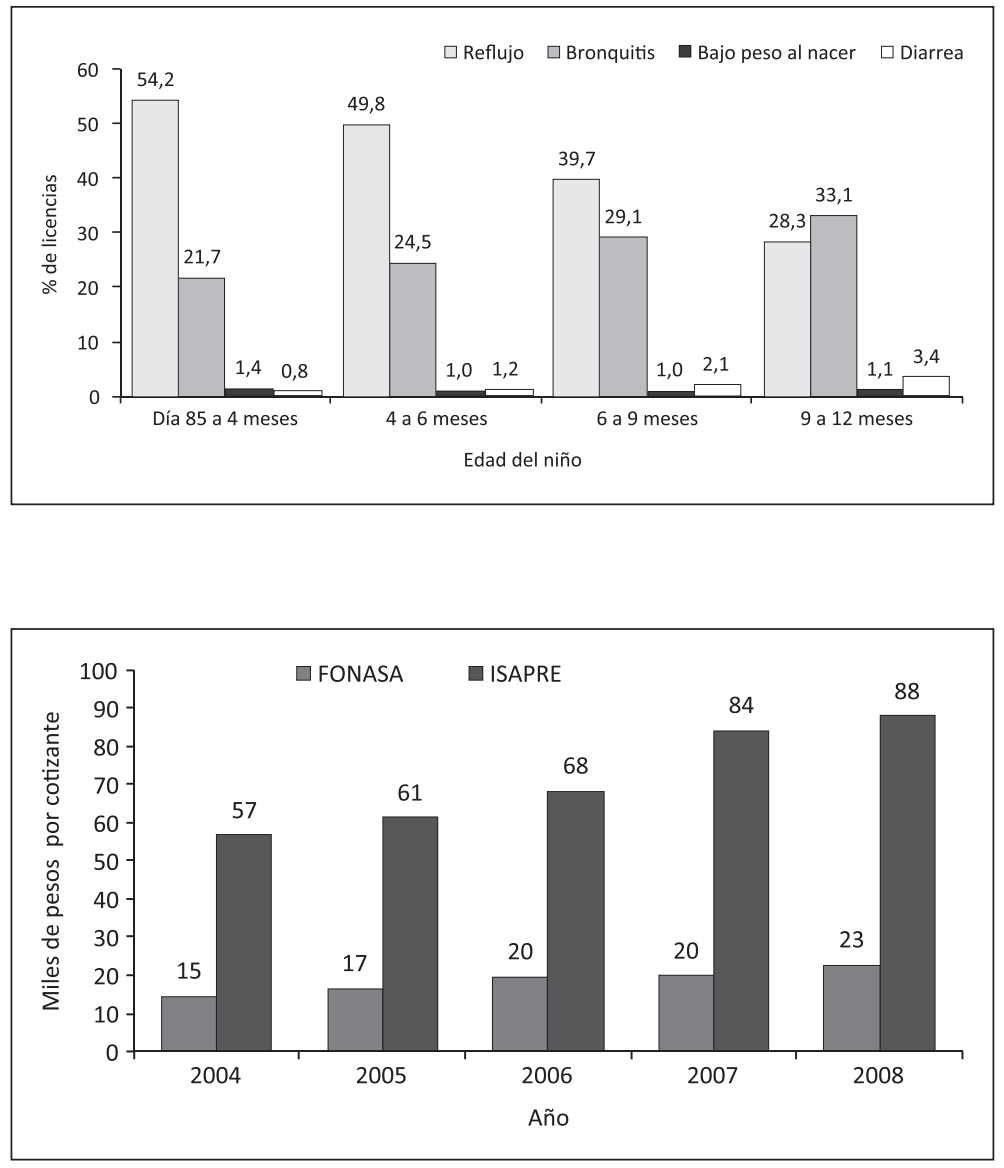

Figura 6. Distribución porcentual del diagnóstico de la licencia según edad del niño(a), para FONASA años 2004-2008. Fuente: Elaboración propia en base a datos de FONASA.

Figura 7. Gasto promedio de licencias EGNMA por mujer cotizante, según FONASA e ISAPRE, años 2004-2008 (Miles de pesos a diciembre 2009). Fuente: Elaboración propia en base a datos de FONASA y Superintendencia de Salud. *Denominador: Mujeres cotizantes en edad fértil (15 a 49 años).

\section{Discusión}

Actualmente existe consenso en ampliar la extensión del postnatal, lo cual es particularmente meritorio considerando que las licencias por EGNMA en la gran mayoría de los países no son pagadas, el subsidio pre y postnatal y el fuero maternal se encuentra entre los más extensos del mundo (Tabla 2) $)^{10-13}$.

Algunos sectores han expresado preocupación por el uso presuntamente abusivo de este subsidio y el efecto que podría tener la ley de extensión de postnatal, actualmente en trámite de promulgación (4 octubre de 2011), sobre la participación laboral de la mujer que es ya reducida en Chile, muy por debajo del promedio de la Organización para la Cooperación y el Desarrollo Económico (OCDE), y también por debajo de países de nivel de desarrollo similar en la región ${ }^{14}$.
El presente estudio confirma el aumento de la utilización y del gasto en las licencias por EGNMA en el período observado, lo que es coincidente con hallazgos de otros estudios ${ }^{1-3}$.

Todos los indicadores analizados son mayores para las trabajadoras que cotizan en el sistema privado. Sin embargo, en todos ellos el aumento es superior para las mujeres cotizantes de FONASA. Una de las razones que probablemente explica este incremento es que no se cuenta con herramientas legales para fiscalizar, dado que su rol se limita a financiar los subsidios pagados por los Servicios de Salud y a cubrir los déficit en que incurren las Cajas de Compensación de Asignación Familiar. Por otra parte, en el marco institucional actual de las ISAPRES no hay incentivos para fiscalizar el gasto de licencias por EGNMA ${ }^{12}$.

Los análisis muestran que no existe una correspondencia entre las principales causas de mortali- 
Tabla 2. Comparación entre distintos países de la duración de los subsidios por reposo maternal (en número de días)

\begin{tabular}{|c|c|c|c|c|c|}
\hline País & Total & Prenatal & Postnatal & Fuente Financiamiento & $\begin{array}{l}\text { Duración licencia } \\
\text { pagada para padres }\left({ }^{*}\right)\end{array}$ \\
\hline \multicolumn{6}{|l|}{ América } \\
\hline Argentina & 90 & 45 & 45 & Seguridad social & Menos de 2 semanas \\
\hline Brasil & 120 & $>=28$ & $>=28$ & Seguridad social & Menos de 2 semanas \\
\hline Chile* & 126 & 42 & 84 & Seguridad social & 5 días \\
\hline Costa Rica & 120 & 30 & 90 & Seguridad social & No se paga \\
\hline México & 84 & 42 & 42 & Seguridad social & No se paga \\
\hline Perú & 90 & 45 & 45 & Seguridad social & 4 o más semanas \\
\hline EE.UU & 84 & & & No pagada & No se paga \\
\hline \multicolumn{6}{|c|}{ Asia y El Pacífico } \\
\hline Australia & & & & No pagada & 4 o más semanas \\
\hline Japón & 98 & 43 & 56 & Seguridad social & 1 a 2 semanas \\
\hline Nueva Zelandia & 98 & $<=42$ & $<=56$ & No pagada & 4 o más semanas \\
\hline \multicolumn{6}{|l|}{ Europa } \\
\hline Alemania & 98 & 42 & 56 & Seguridad social y empleador & 4 o más semanas \\
\hline Bélgica & 105 & 49 & 56 & Seguridad social & 4 o más semanas \\
\hline Dinamarca & 126 & 28 & 98 & Seguridad social & 4 o más semanas \\
\hline España & 112 & $<=70$ & $>=42$ & Seguridad social & 4 o más semanas \\
\hline Finlandia & 105 & $<=50$ & $>=30$ & Seguridad social & 4 o más semanas \\
\hline Francia & 112 & 42 & 70 & Seguridad social & 4 o más semanas \\
\hline Italia & 150 & 60 & 90 & Seguridad social & 3 a 4 semanas \\
\hline Noruega & 126 & $<=84$ & $>=42$ & Seguridad social y empleador & 4 o más semanas \\
\hline Rumania & 112 & 52 & 60 & Seguridad social & 3 a 4 semanas \\
\hline Suecia & 84 & 42 & 42 & Seguridad social & 4 o más semanas \\
\hline
\end{tabular}

Fuente: Elaboración propia en base a Aedo Cristián, "Evaluación Económica de la Prolongación del postnatal". Rev Chil Pediatr 2007, y Raising the global floor: Adult Labour. A Word Legal Rights Database. *A septiembre 2011.

dad o morbilidad infantil y el aumento sostenido de las licencias en este grupo de población? ${ }^{7}$.

Se observa inconsistencia entre los diagnósticos más frecuentes asociados al otorgamiento de las licencias por EGNMA y el perfil de prevalencia de dichas enfermedades. El caso emblemático lo constituye el reflujo gastro-esofágico que el año 2008 representa el 58\% de licencias de ISAPRE y $50 \%$ de FONASA. Esto contrasta con la prevalencia de 8 a 12\% de reflujo encontrada en estudios internacionales ${ }^{15,16}$. Además se observa que hay una concentración de casi 35\% de las licencias por EGNMA inmediatamente terminado el post natal y casi $60 \%$ antes de los 6 meses.
Lo anterior permite plantear que posiblemente este subsidio se está utilizando como una manera de prolongar el período de reposo laboral postnatal.

Acerca de los incentivos para un sobreuso, hay antecedentes que sustentan que ellos podrían estar presentes. En primer lugar este subsidio se financia con impuestos generales, sin carga financiera para los seguros ni para las trabajadoras, lo que podría explicar la baja tasa de rechazo de estos subsidios (alrededor de 6\%), y la baja frecuencia de reducción de días (3,5\%), comparado con más de 12\% en ambos subsistemas para el caso de licencias curativas en que el pago si repercute en los seguros, 
en segundo lugar la decisión del subsidio depende del profesional médico en un escenario de baja regulación de la utilización adecuada.

Desde la perspectiva de equidad, se verifica que la utilización actual de este subsidio, es regresiva, ya que beneficia en primer lugar sólo a las mujeres que trabajan y cotizan en algún sistema previsional de salud, y lo que es más preocupante, es utilizado principalmente por las mujeres pertenecientes al sistema privado, que según datos de la encuesta CASEN 2009, ganan 2,7 veces más que las mujeres del sistema público ${ }^{9,17}$. Adicionalmente, la brecha del monto pagado por licencias EGNMA al año a las cotizantes es 4 veces mayor en el sistema privado comparado con el público.

La nueva ley entregará a las beneficiarias 12 semanas adicionales de post-natal, las cuales pueden ser traspasadas al padre a partir de la $7^{\mathrm{a}}$ semana, pagándose el subsidio en base al salario de quien haga uso de él. En cuanto a las licencias por EGNMA mantiene los actuales criterios de que sea el médico el que define el carácter de enfermedad grave. Además se amplía el número de mujeres que tienen derecho al beneficio incorporando a las trabajadoras sin contrato de trabajo que integren un hogar perteneciente al $60 \%$ más pobre de la población, siempre que tengan al menos doce meses de afiliación previsional antes del embarazo y ocho o más cotizaciones continuas o discontinuas en los 24 meses anteriores al embarazo.

Desde el punto de vista sanitario, los efectos de estos cambios serán sin duda beneficiosos. En primer lugar, se disminuye la regresividad del subsidio al incorporar a las mujeres más vulnerables al beneficio. En segundo lugar permite a las madres y padres la posibilidad de permanecer junto a sus hijos hasta los seis meses de edad, lo que tendría efectos positivos en el apego. La incorporación del padre al beneficio del cuidado del hijo es un aspecto favorable adicional en términos del vínculo padre-hijo, además se reparte de manera más equitativa el costo laboral de la maternidad entre la madre y el padre.

Se propone para futuras investigaciones dar seguimiento y profundizar en el tema analizando por ejemplo, el impacto de la implementación de esta ley en la evolución de las licencias por EGNMA post implementación.

Finalmente, en relación a limitaciones de este estudio, la principal, dice relación con la ausencia de información en la variable diagnóstico que al- canza al 5,4\% de las licencias emitidas de FONASA, mientras que para ISAPRE es $0,5 \%$.

Esta situación se presenta también en la variable fecha de nacimiento del niño, en la cual la falta de información es de 10,7\% para FONASA. En ISAPRE no se contó con esta información.

Agradecimientos. A la División de Investigación de la Universidad del Desarrollo por el apoyo a la implementación de esta línea de investigación. Al Fondo Nacional de Salud y a la Superintendencia de Salud por facilitar las bases de datos. Al Señor Vicente Zúñiga M. por su contribución en la edición de este documento.

\section{Referencias}

1. Pezoa M. Licencias Médicas-Gasto por Subsidio por Incapacidad Laboral (SIL), Superintendencia de Salud, Departamento Estudios y Desarrollo, Chile. 2010. Disponible en: http://www.supersalud.cl (consultado el 29 de diciembre de 2010).

2. Delgado I, Zúñiga V. Estudio del Gasto por Subsidio de Incapacidad Laboral de Cotizantes FONASA e ISAPRE en los últimos 5 años. Ministerio de Salud, MINSAL, Chile, 2010. En edición.

3. Tokman M, Rodríguez J, Larraín F. Subsidios por incapacidad laboral 1991-2002: Incentivos institucionales, crecimiento del gasto y una propuesta de racionalización. Centro de Estudios Públicos, Chile. Documento de Trabajo no93, 2004. Disponible en: www.cepchile.cl (consultado el 10 de noviembre de 2010).

4. Código del Trabajo de la República de Chile, Dictámenes y Normativas, Libro II De la protección a los trabajadores, Título II De la protección a la maternidad, Art 194-208. Código del Trabajo. Dirección del trabajo. Disponible en:www.dt.gob.cl (consultado el 10 de noviembre de 2010).

5. Kosmala-Anderson J, Wallace L. Breastfeeding works: the role of employers in supporting women who wish to breastfeed and work in four organizations in England. J Public Health 2006; 28 (3): 183-91.

6. Guendelman S, Lang J, Pearl M, Graham S, Goodman J, Kharrazi M. Juggling Work and Breastfeeding: Effects of Maternity Leave and Occupational Characteristics. Pediatrics 2009; 123 (1): e38-e46.

7. Vargas N, Guezada A. Epidemiología, nueva morbilidad pediátrica y rol del pediatra. Rev Chil Pediatr 2007; 78 (suppl. 1): s103-10.

8. Dussaillant F. ¿Más salas cuna o permisos post natales 
Licencia por enfermedad grave del niño(a) menor de un año: certezas e interrogantes - I. Delgado et al

más largos? Una comparación de alternativas de política para apoyar A la maternidad y a la primera infancia. Centro de Estudios Públicos, Chile. Documento de trabajo no 377, 2009. Disponible en: www.cepchile.cl (consultado el 23 de diciembre de 2010).

9. Cariola MG, Bedregal P, Bell A, Cerda R, Domínguez C, Carey S, et al. Mujer, Trabajo y Maternidad. Comisión Asesora Presidencial, Chile. 2010. Disponible en: http:// www.comisionmujertrabajoymaternidad.cl/ (consultado el 10 de noviembre de 2010).

10. Aedo C. Evaluación económica de la prolongación del postnatal. Rev Chil Pediatr 2007; 78 (Supl 1): 10-50.

11. McGill Institute for Health and Social Policy. Raising the global floor: Adult Labour. A Word Legal Rights Database. Disponible en: http://raisingtheglobalfloor. org/policies/policy-selection.php?policy=paidleave (consultado el 13 de septiembre de 2011).

12. Rodríguez J, Tokman M. Licencias médicas: ¿Chilenos enfermizos? Expansiva, Serie en foco no 16, Chile. 2003.

13. Organización Internacional del Trabajo (OIT). La ma- ternidad en el trabajo: examen de la legislación nacional. Oficina Internacional del Trabajo, Condiciones de Trabajo. 2a edición. Ginebra; OIT, 2010. Disponible en: http://www.expansiva.org/ (consultado el 10 enero de 2011).

14. Cifuentes C. Extensión del Postnatal: Análisis del Proyecto de Ley. Libertad y Desarrollo, Serie Informe Económico no 215, Chile. 2011.

15. Campanozzi A, Boccia G, Pensabene L, Panetta F, Marseglia A, Strisciuglio P, et al. Prevalence and Natural History of Gastroesophageal Reflux: Pediatric Prospective survey. Pediatrics 2009; 123(3): 779-83.

16. Ramírez J, Mata N, Cervantes R, Zárate F, Munguía P, López $\mathrm{G}$, et al. Reflujo gastroesofágico y asma de reciente diagnóstico en niños. Alergia e Inmunología Pediátrica 2001; 10 (3): 77-81.

17. Ministerio de Planificación (MIDEPLAN). Encuesta de Caracterización Socioeconómica Nacional, CASEN 2009. Disponible en: http://www.mideplan.gob.cl/casen2009/ (consultado el 2 diciembre de 2010). 